\section{MAGNETIC FIELD CONTROLLED CAPACITOR}

\section{Petr Polcar - Daniel Mayer}

Abstract - this paper deals with the capacitor using magnetic fluid as a magnetic field controlled dielectrics. It is shown, that dielectrics of this capacitor exhibits magnetic field induced anisotropy.

Keywords: magnetic fluid, magnetic field controlled capacitor, dielectric anisotropy

\section{INTRODUCTION}

A capacitor using a magnetic fluid as a dielectric is presented in this paper. The capacity of this capacitor can be controlled with an external magnetic field, as shown in Fig. 1.

Magnetic fluids represent relatively new electrical material; first stable fluid was synthesized in 1964. The research of magnetic fluids was under embargo at first, but in 70ties of last century, the results were declassified and a large number of journal and book has appeared since ( $e g$ there is a list of 544 citations in [1] and 652 citations in [2]) and many useful applications of magnetic fluids were patented.

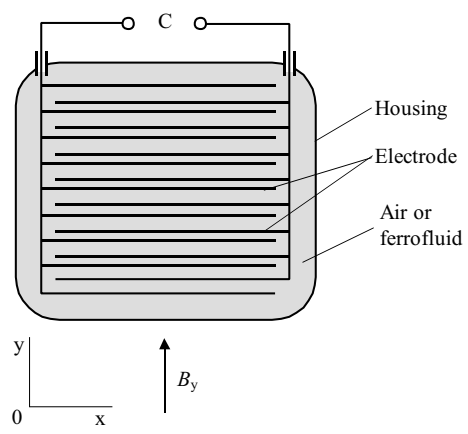

Fig. 1. Capacitor with magnetic fluid

Magnetic fluid generally consists of permanently stable, electrically non conductive suspension of magnetic dipolar particles $\left(e g \mathrm{Fe}_{3} \mathrm{O}_{4}\right)$ in carrier liquid. Based on the size of these particles, magnetic fluids are divided into ferrofluids ( $5 \sim 15 \mathrm{~nm}$ ) and magnetorheological fluids (in order of units and tens of $\mu \mathrm{m})$. To prevent the aggregation, the particles are covered with a macromolecular polymeric sheet, so called detergent (sometimes listed as surfactant). Various oils, often based on hydrocarbon, are most commonly used as a carrier liquid.

Without the effect of an external magnetic field, ferromagnetic particles are oriented randomly in the liquid. The external magnetic field causes the particles to group into chains. These chains copy the course of the magnetic field lines of force. These structural changes cause that the magnetic fluid ceases to behave as an isotropic medium and becomes an anisotropic material, this effect is called magnetic induced anisotropy [3]. Macroscopically, physical properties of the liquids change. The dominant property of magnetorheological fluids is their viscosity change, the magnetorheological effect, which is used in several technical applications, eg magnetic field controlled dampers, or crankshaft seals $[4,5]$.

\section{THE PHYSICOCHEMICAL NATURE OF THE MAGNETIC FIELD CONTROLLED CAPACITOR}

Without an external magnetic field affecting the fluid, the ferromagnetic particles are randomly organized and the magnetic fluid behaves as an isotropic dielectric, which can be characterized by a scalar variable - the permittivity $\varepsilon=\varepsilon_{o} \varepsilon_{r}$, where $\varepsilon_{o}=8.85 \times 10^{-12} \mathrm{Fm}^{-1}$ and $\varepsilon_{r}$ is the relative permittivity.

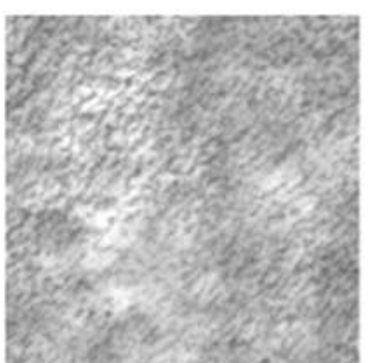

(a)

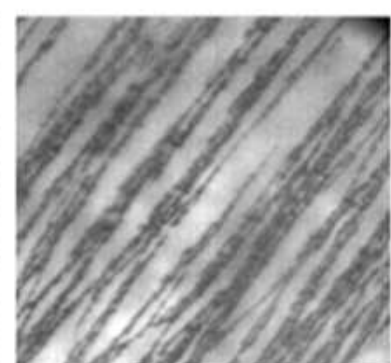

(b)
Fig. 2. Photomicrograph of particles of $\mathrm{Fe}_{3} \mathrm{O}_{4}$ of magnetic fluid MRHCCS4-B without and with the external field

The external magnetic field causes structural changes and the fluid becomes anisotropic (Fig. 2) and when properly established cartesian (Fig. 3), can be characterized with the permittivity tensor

$$
\varepsilon=\varepsilon_{0}\left[\begin{array}{cc}
\varepsilon_{r x} & 0 \\
0 & \varepsilon_{r y}
\end{array}\right]
$$

* Department of the Theory of Electrical Engineering, Faculty of Electrical Engineering, University of West Bohemia in Pilsen, Univerzitni 8, 30614 Pilsen, polcarp@kte.zcu.cz, mayer@kte.zcu.cz 


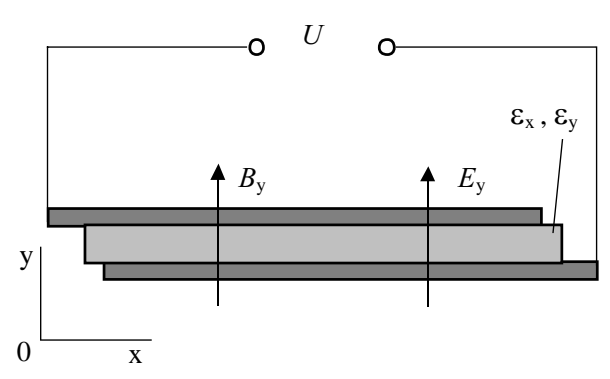

Fig. 3. Magnetic field controlled capacitor cartesian establishment

while $\varepsilon_{r x}, \varepsilon_{r y} \neq \varepsilon_{r}$. This effect will be called magnetic field induced dielectric anisotropy - MFIDA.

While without the external magnetic field effect is the capacity of the capacitor $C_{0}=C\left(\varepsilon_{r}\right)$, with the effect of the external field $B_{y}$ is its capacity $C=C\left(\varepsilon_{r y}\right) \neq C_{0}$. The magnetic induction of the external field $B_{y}$ determines the particles chaining rate (till the saturation) and with it the magnitude of the permittivity tensor components $\varepsilon_{r x}, \varepsilon_{r y}$, therefore the capacity of device is $C=C\left\{\varepsilon_{r y}\left(B_{r}\right)\right\}$.

The arrangement of ferromagnetic particles, therefore the value of capacity $C$, is generally dependant on the intensity of the electric field $E_{y}$ as well. Until now, no voltage $U$ on the capacitor electrodes was considered. If $U \neq 0$, the arrangement of the fluid structure (and its permittivity) is affected by the intensity $E_{y}$ as well, the capacitor with liquids dielectric is nonlinear consequently. This effect will be called electric field induced dielectric anisotropy - EFIDA. Both of these phenomena, MFIDA and EFIDA, act simultaneously. In experiments executed by us, the effect of EFIDA was negligible therefore $C(U) \sim$ const.

Due to the ferromagnetic particles weight and the viscosity of the carrier liquid, mechanical dynamics applies when ferromagnetic particles chains are created and the chaining shows certain time delay. This is apparent from the time sequence of photomicrographs showed in Fig. 4.

\section{DESIGN OF EXPERIMENTAL SAMPLES}

An experimental multilayer planar capacitor was made with the use of a 3D printer (A sketch of one layer can be seen in Fig. 5., the scheme of composition in the Fig. 6.)

The electrodes are made from an aluminium sheet and linked together with copper conductors. The area between electrodes was filled with the magnetic liquid MRHCCS4B provided by the Liquids research, Ltd. The thickness of the layers was chosen in such a way that no mechanical sag of electrodes causing the change of capacity can occur. The housing and non conductive parts of the device were printed from plastic (concretely ABS) and two supplies are lead out of the capacitor. To prevent incidental leak of the fluid, the outside of the device was cemented and varnished.

\section{EXPERIMENTS}

A common alternate bridge was used to measure the capacity of the controlled capacitor prototype. To verify the measured values, the capacity of the device was determined from the time response to its charging as well to eliminate appropriate dynamic changes caused by the current powering the bridge. The time of this response was intentionally prolonged by a serial resistance. Result obtained by both methods were closely comparable.

\section{1 $C=f(B)$ characteristics}

The capacitor prototype was placed together with the Hall probe of teslameter Elimag MP-1 between the poles of (previously demagnetised) magnetic circuit and connected to the RLC measuring bridge U1733C. Gradually increasing the powering current to the magnetic circuit,
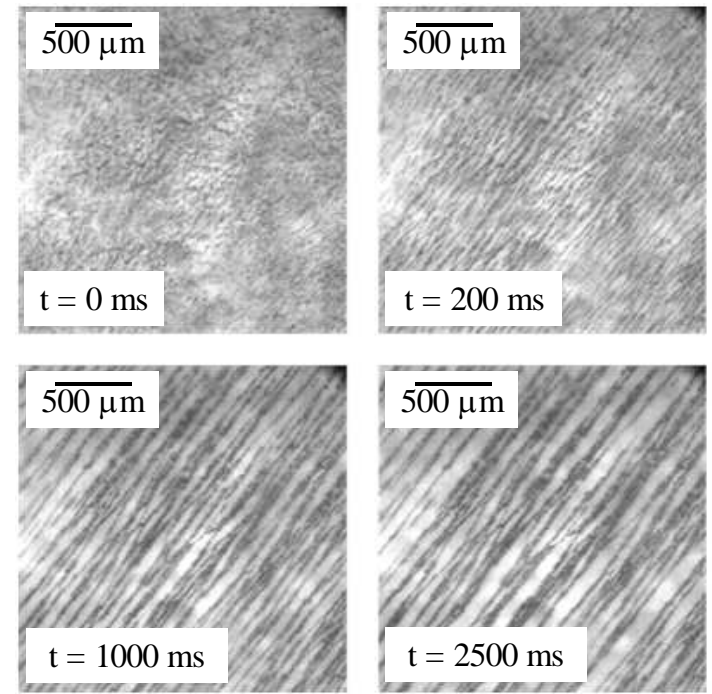
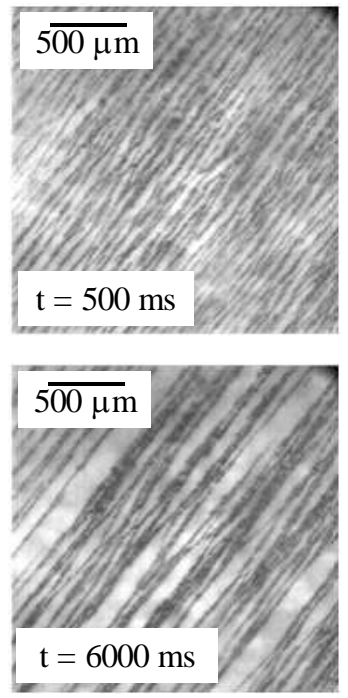

Fig. 4. High speed camera photomicrograph, the time delay of the MRHCCS-4B fluid ferromagnetic particles chaining is apparent. The external field $B_{y}=22 \mathrm{mT}$ was applied on the fluid at the time $t=0$ 
(a)

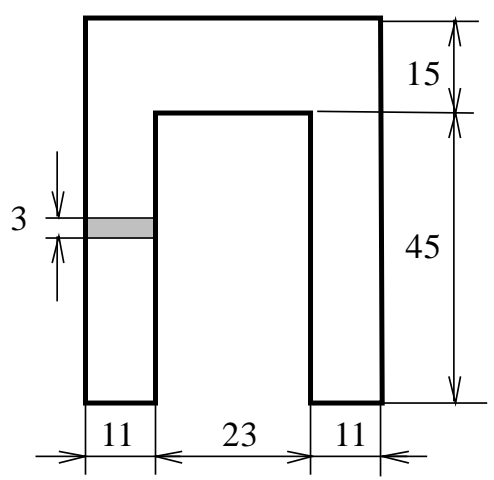

(b)

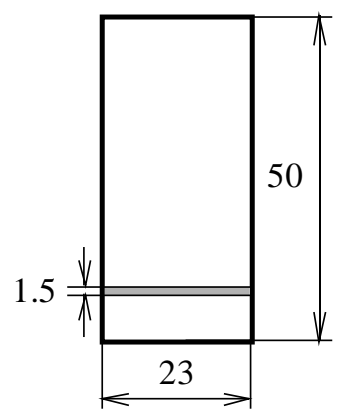

(c)

Fig. 5. Dimensions of the capacitor layer; (a),(b),(c) — plastic support aluminium electrodes?

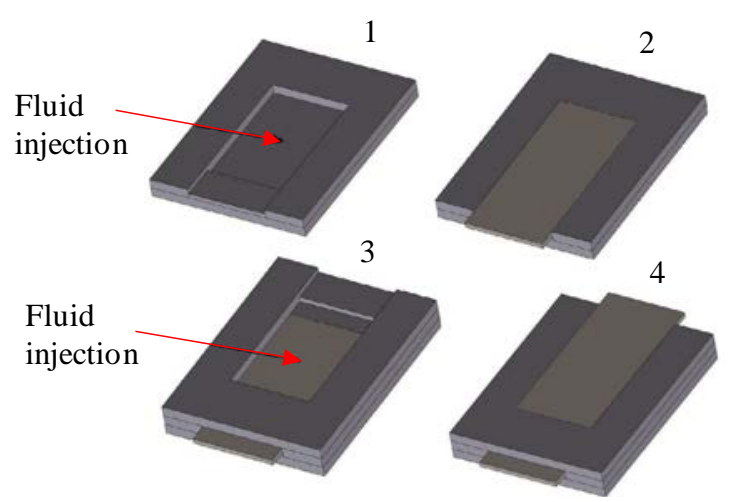

Fig. 6. Composition of two layers of the multi layer capacitor prototype

external magnetic field effecting the capacitor $B_{y}$ rises, values of $B_{y}$ and $C$ were registered. To examine the dynamics of the micro structural changes of the used fluid, the magnetic field was gradually incremented with a constant time step between individual increments. To investigate the hysteresis, the magnetic field was gradually low-

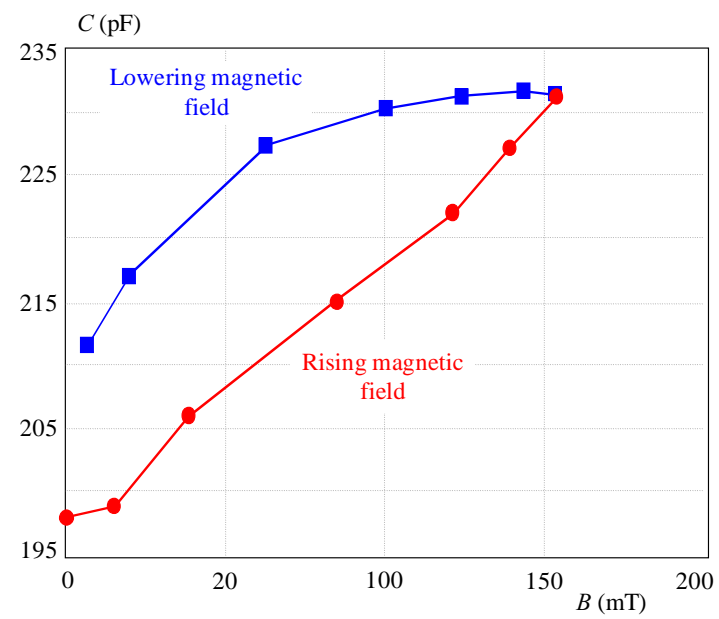

Fig. 7. Hysteresis of the dependence $C(B)$ of the measured capacitor with magnetic fluid. The time interval between individual measurements was $\Delta T=30 \mathrm{~s}$ ered after, again with a constant time step. Measured characteristics can be seen in Figs. 7 and 8 .

Time to reach the steady state of the capacity of the measured capacitor $\mathrm{C}$, after removing the external magnetic field, is in order of minutes.

\subsection{Leakage current}

No leakage current was measured when the capacitor was long-term connected to a $\mathrm{DC}$ voltage with value $U_{0}=100 \mathrm{~V}$. Ammeter with minimal current resolution of $I_{\min }=1 \mu \mathrm{A}$ was used. No current was measured even when the capacitor was placed in the external magnetic field of $B_{y}=0.3 \mathrm{~T}$ (This field was generated with the use of a permanent magnet.).

\subsection{Measuring of EFIDA, ie $C=f(U)$ character- istics}

The characteristics of $C=f(U)$ was constructed from the gradual charging of capacitor after it was connected to a DC voltage source. The voltage $U_{0}$ was jump in individual steps and generated transient was measured with an oscilloscope. To slower the time response, a resistor

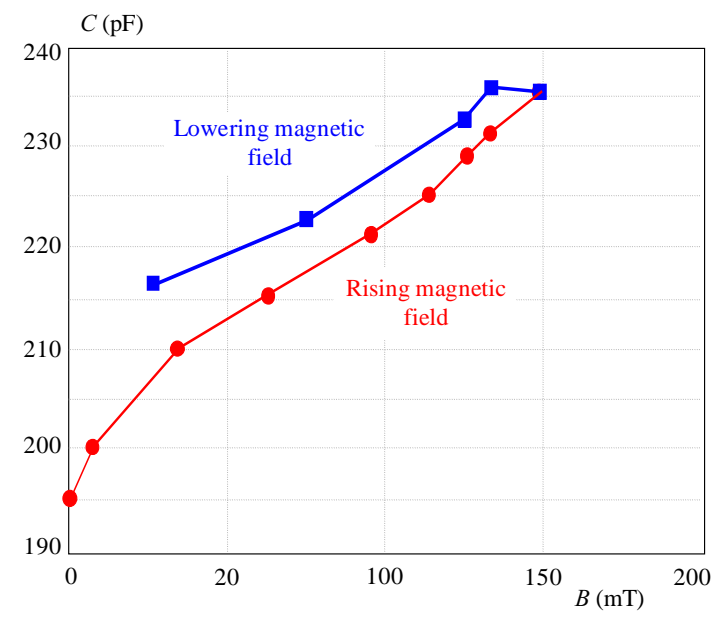

Fig. 8. Hysteresis of the dependence $C(B)$ of the measured capacitor with magnetic fluid. The time interval between individual measurements was $\Delta T=60 \mathrm{~s}$ 
$R=33 \mathrm{k} \Omega$ was serially connected to the measured capacitor. From the relation for the time constant $\tau=R C$, values of the capacity were determined after, while the time to reach the value of $U_{C}=0.63 U_{0}$ was taken for the time $\tau$. When changing the voltage $U_{0}$ in the interval $\langle 0.5 ; 4 \mathrm{~V}\rangle$, the capacity with deviations $C=198 \pm 5 \mathrm{pF}$ with random distribution was measured. These deviations were considered to be the error of the used method for determining the capacity, not for the evidence of EFIDA phenomena $C=f(U)$.

\section{CONCLUSION}

Interesting phenomena in magnetic fluids, MFIDA, res. EFIDA, during which the isotropic magnetic fluid under the influence of the external magnetic, resp. electric field, changes to anisotropic medium were highlighted. First of this phenomena was used to design a capacitor, whose capacity can be changed with the use of an external magnetic field.

The external magnetic field induces structural changes in the magnetic fluid and because of the weight of the ferromagnetic particles chains are time responses (ie capacity change, token of hysteresis) relatively slow, in order up to minutes. In addition, another attribute of magnetic liquids is the instability of their physical properties ( $e g$ effect of the ambient temperature, hydroscopicity, evaporation, and other) and with it the low accuracy in reproducing investigated phenomena, which apparently lowers the possible applicability of the designed controlled capacitor, especially eg for measurement purposes. The authors were not able to perform measurements on the capacitor for different types of magnetic fluids, but because of nowadays wide range of these materials, the existence of such fluid is possible, that exhibits even stronger capacity change and sensitivity, than in experiments described. Despite listed limitations we assume, that this, by our opinion innovative application of magnetic fluids, deepens the existing knowledge of the magnetic fluids and the designed controlled capacitor posses advantageous properties as well, e.g. simplicity and with it connected reliability, low price etc and it will find its practical uses, for example in sensors detecting the changes of the magnetic field.

\section{Acknowledgement}

The support of the University of West Bohemia in Pilsen, namely research project SGS-2015-035, is gratefully acknowledged.

\section{REFERENCES}

[1] BERKOvSKI, B.-RASHTOVO, V. (Eds): Magnetic Fluids and Application, Handbook, Regel House, New York, 1996.

[2] BUtTeR, K.-BOMAns, P. H. H.-FREDERIK, P. M.VROEGE, G. J.-PHILIPSA, A. P.: Direct Observation of Dipolar Chains in Iron Ferrofluids by Cryogenic Electron Microscopy, Nature Materials 2 (Feb 2003), 88-91.

[3] MAYER, D.: An Approach to Measurment of Permeability/Permittivity Tensor of Ferrofluids, Journal of Electrical Engineering 66 No. 5 (2015), 292-296.

[4] ODENBACH, S. : Magnetoviscous Effects in Ferrofluids, Springer-Verlag, Berlin, 2002.

[5] KUBÍK, M.-MAZŮREK, I.-ROUPEC, J.-STRECKER, Z.-MACHÁČEK, O.: Design of Semi-Active Magnetorheological Valve with Non-Magnetic Bypass, Transactions on Electrical Engineering No. 1 (2015), http://www.transoneleng.org, [Accessed: 16 Nov 2015].

[6] KÚDELČÍK, J.-BURY, P.-KOPČANSKÝ, P.-TIMKO, M.-ZÁVIŠOVÁ, V.: Acoustic Properties of Magnetic Fluids based on Transformer Oil under Magnetic Field, Journal of Electrical Engineering 64 No. 6 (2013), 381-385.

[7] CHANTRELL, R. W.-BRADBURY, A.-POPPLEVELL, J. - CHARLES, S. W.: Agglomeration Formation in Magnetic Fluid, J. Appl. Phys. 53 No. 3, 2742-2744.

[8] MAYER, D.: Future of Electrotechnics: Ferrofluids, Advances in Electrical and Electronic Engineering 7 No. 1-2 (2008), 9-14.

[9] ODEnBACH, S. (Ed): Colloidal Magnetic Fluids, Springer-Verlag, Berlin, 2009.

Received 11 November 2015

Petr Polcar (1983). He graduated (Ing) in 2007, in 2014 he defended his $\mathrm{PhD}$ thesis in the field of electromechanical systems using magnetic liquids. He works at the Department of Theory of Electrical Engineering at the University of West Bohemia in Pilsen. His scientific research aims on applied electromagnetic field theory with main focus on electromagnetic properties and applications of magnetic liquids.

Daniel Mayer, Prof, Ing, DrSc received the Ing, $\mathrm{PhD}$ and DrSc degrees in electrical engineering from Technical University in Prague, Czech Republic. In 1959 Associate Professor at the University of West Bohemia in Pilsen, in 1968 full Professor of the Theory of Electrical Engineering. Many years he was head of the Department of Theory of Electrical Engineering. Research interests: circuit theory, electromagnetic field theory, electrical machines and apparatus, history of electrical engineering. He published 7 books, more than 330 scientific papers and 10 patents. He is a member of editorial boards of several international journals and leader of many grant projects. 